\title{
Muttersprachliche psychiatrische Behandlung
}

\author{
Psychiatric Therapy in the Native Language
}

Autoren

Pro: Yesim Erim

Kontra: Eckhardt Koch

Bibliografie

Dol http://dx.doi.org/

10.1055/s-0030-1265959

Psychiat Prax 2011; 38: 5-7

(c) Georg Thieme Verlag KG

Stuttgart · New York .

ISSN 0303-4259

Korrespondenzadressen

Priv.-Doz. Dr. Yesim Erim

Leitende Oberärztin, Klinik für

Psychosomatische Medizin und

Psychotherapie am Universitäts-

klinikum Duisburg-Essen

Virchow-Straße 174

45147 Essen

yesim.erim@uni-due.de

\section{Dr. Eckhardt Koch}

Leitender Arzt Interkulturelle Psychiatrie und Migrationsbeauftragter

Vitos Klinik für Psychiatrie und Psychotherapie Gießen-Marburg Cappeler Straße 98

35039 Marburg eckhardt.koch@vitos-giessenmarburg.de

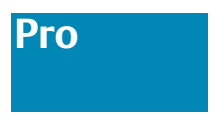

Parallel zur Einrichtung von Migrantenambulanzen in psychiatrischen Institutionen und von spezialisierten stationären Behandlungssettings für Migranten in psychosomatischen Kliniken entstand Mitte der 90er-Jahre der Begriff „muttersprachliche Behandlung“. Gemeint ist der Einsatz von bilingualen Mitarbeitern mit psychiatrischer und psychotherapeutischer Fachkompetenz in der Versorgung von Migranten. Meistens handelt es sich um Kinder von Gastarbeitern, die neben ihrer beruflichen auch ihre kulturelle und sprachliche Kompetenz in ihre Tätigkeit einbringen. Die muttersprachliche Behandlung ist eine Antwort der psychiatrischen und psychosomatischen Institutionen auf die besonderen Bedürfnisse der zunehmenden Population mit Migrationshintergrund.

In der Literatur finden wir 2 ähnliche Begriffe: Kirmayer [1] definiert den kulturellen Konsiliardienst (Cultural consultation service) und Tantam [2] beschreibt die „ethnische Passung“ (=ethnic matching), die Behandlung der Migranten durch Therapeuten aus der gleichen ethnischen Gruppe. Unter die Aufgaben des kulturellen Konsiliardienstes subsumiert Kirmayer eine ausführliche Erstdiagnostik durch einen muttersprachlichen Experten bei Zuweisung von Patienten mit kulturellen oder sprachlichen Besonderheiten. Dabei sollen die für die Diagnostik und Therapie relevanten kulturellen Besonderheiten benannt werden. Auch die Beratung von autochthonen (einheimischen) Therapeuten bei schwierigem Behandlungsverlauf und die Untersuchung kulturspezifischer Begebenheiten, die den Therapieverlauf beeinflussen, gehören zum Aufgabenspektrum eines solchen Konsiliardienstes.

Was sind die Indikationen für eine muttersprachliche psychiatrische Behandlung?

Die kultursensitive Befunderhebung (cultural formulation), die in DSM-IV eingeführt wurde, hat weltweit wenig Verbreitung gefunden. Mezzich et al. [3] erklären diesen Umstand mit dem Fehlen geeigneter Fallbeispiele und empfehlen in ihrer Leitlinie für kultursensitive Befunderhebung (Cultural Formulation Guidelines) folgende 4 Bereiche zu fokussieren:

1. Kulturelle Identität des Patienten einschließlich seiner Wertorientierungen, Sprachkenntnisse, Krankheitskonzepte, Selbst- und Körperbild, Weltanschauung,

2. Einflüsse der Kultur auf das Krankheitserleben und das Inanspruchnahmeverhalten sowie

3. auf die Interaktion mit der Familie und schließlich

4. auf die Interaktion zwischen dem Patienten und dem Untersucher.

Durch die formulierten Forderungen an eine kultursensitive Diagnostik ist die Frage der Indikation für eine muttersprachliche psychiatrische Behandlung teilweise schon beantwortet. Sie ist immer dann indiziert, wenn die Kenntnisse des Patienten nicht ausreichen, emotionale und kognitive Zusammenhänge in der Sprache des Aufnahmelandes zum Ausdruck zu bringen. Der muttersprachliche Diagnostiker kann in vielen Fällen sicherer beurteilen, ob z.B. bestimmte sprachliche Äußerungen („Meine inneren Organe brennen“: Melancholischer Wahn oder kultureller Sprachgebrauch?) oder ein bestimmtes Verhalten (Patient nimmt Medikamente nicht ein und lässt sich von einem religiösen Heiler behandeln: Wahn oder kulturell geprägter Heilungsversuch?) einer kulturellen Haltung oder der vorliegenden psychischen Störung zuzuschreiben sind.

Wirkfaktoren und Effekte der muttersprachlichen psychotherapeutischen Behandlung

Der Einfluss der Kultur auf unser Erleben und Verhalten wird in der psychotherapeutischen Behandlung von Migranten sehr deutlich. Angestrebte Veränderungen sind meist nicht nur indi- 
vidueller Natur, sondern sie kollidieren oft mit grundlegenden kulturellen Werten, die bisher leitend waren [4].

Migranten können aus einer Kultur kommen, die Hilfebeziehung nicht im Zweiergespräch, sondern z. B. in gemeinsamen Anstrengungen von Familien oder Freunden ansiedelt. Sie können vielfältige Diskriminierungen im Aufnahmeland erfahren haben. In diesen Fällen werden sie in der klassischen einzeltherapeutischen Situation und gegenüber einem Therapeuten, den sie als Vertreter der Dominanzkultur wahrnehmen, verunsichert sein. Die „Anfangssympathie“ der Klienten zu gewinnen und zu planen, welche Haltungen für die Erstellung einer therapeutischen Allianz hilfreich sind, ist in der Behandlung von Migranten sehr wichtig. In der muttersprachlich-bilingualen Psychotherapie haben die Migranten eine größere Zuversicht darüber, mit ihrer Andersartigkeit Akzeptanz zu finden.

Die muttersprachliche Behandlung hat Ähnlichkeiten mit dem Prinzip des „Peer Counseling“, das im Rahmen der Bewegung „Selbstbestimmtes Leben“ der Behinderten in den USA entstand. Dahinter verbirgt sich die Erfahrung aus Selbsthilfegruppen, in denen Personen in einer ähnlichen schwierigen Lebenssituation einander unterstützen und sich gegenseitig austauschen. Dieser Austausch führt zu einer bewussteren Erfahrung der eigenen Identität.

Tantam [2] vertritt die These, dass ein Behandler gegenüber einem Patienten aus der gleichen Ethnie eine bessere oder promptere Empathie entwickeln kann, wenn man die empathische Einfühlung als eine emotionale Ansteckung versteht, die von bisherigen Beziehungserfahrungen und -erwartungen genährt wird. Als relevante Argumente für muttersprachliche oder „ethnisch passende“ (ethnic matching) Psychotherapeuten führt sie an, dass durch ihren Einsatz eine höhere Inanspruchnahme der Institutionen durch Migranten sowie eine bessere Therapiemotivation und Compliance der Migranten erreicht werden. Tatsächlich ist auch in Deutschland eine der wichtigsten Erfahrungen in der Versorgung von Migranten die Feststellung gewesen, dass die Inanspruchnahme durch Migranten nach der Etablierung muttersprachlicher Angebote, z.B. sog. „Migrantenambulanzen" rasch und deutlich zunahm $[5,6]$. Durch den Einsatz von muttersprachlichen Fachkräften leisten die Institutionen einen wichtigen Beitrag zum Abbau von Zugangsbarrieren und zur interkulturellen Öffnung [7].

Schließlich stellt die muttersprachliche Behandlung, nicht wie manchmal geschildert, einen Raum dar, in dem die Ursprungskultur idealisiert und eine Abschottung gegenüber der Kultur des Aufnahmelandes unterstützt wird. Vielmehr bietet sie einen „transkulturellen Entwicklungsraum“, so wie von Özbek und Wohlfahrt [8] beschrieben. Sie ermöglicht Patienten, sich in einem geschützten Rahmen mit den Werten der neuen Kultur auseinanderzusetzen und mit diesen zu experimentieren [9].

Die muttersprachliche Behandlung ist eine wertvolle und bisher wichtigste Antwort der deutschen Psychiatrie auf die Bedürfnisse der Migranten. Durch sie kann die Diagnostik verbessert, die Behandlungscompliance und die Inanspruchnahme der Patienten optimiert werden. Schließlich kommt es in Institutionen, in denen muttersprachliche Psychotherapeuten arbeiten, zu einer Sensibilisierung aller Mitarbeiter für kulturelle Besonderheiten.

\section{Kontra}

Der Anteil von Menschen mit Migrationshintergrund beträgt nahezu $20 \%$ der deutschen Bevölkerung. Aktuelle Untersuchungen [10] zeigen, dass die Patienten auch in den psychiatrischen Krankenhäusern in vergleichbarer Zahl ankommen und behandelt werden.

Der Begriff Migrationshintergrund [11] ist sehr weit gefasst und schließt in Deutschland geborene Kinder aus binationalen Ehen ebenso wie erst kürzlich eingereiste Flüchtlinge und Asylsuchende ohne Kompetenz der deutschen Sprache ein. Zwar sind verschiedene Sprachen (z.B. türkisch, arabisch, russisch, polnisch, persisch) recht häufig vertreten, insgesamt leben in Deutschland aber Menschen, die mehr als 150 verschiedene Sprachen sprechen: diese Vielfalt kann nicht mit Konzepten, die auf muttersprachliche Behandlung ausgelegt sind, erreicht werden.

\section{Muttersprachliche Behandlung}

Sicherlich ist es sinnvoll, in Ballungsräumen auch muttersprachliche Versorgungsangebote für zahlenmäßig stark vertretene Ethnien zu etablieren. Gerade in dem unterversorgten Segment ambulanter Psychotherapie für Migranten mit unzureichenden Kenntnissen der deutschen Sprache ist das erforderlich. Allerdings wird hierdurch das generelle Problem einer chancengleichen Behandlung für Menschen mit Migrationshintergrund nicht gelöst. Auch möchte nicht jeder muttersprachlich versierte Therapeut ausschließlich Patienten aus seinem Herkunftsland behandeln, was die geringen Kapazitäten weiter reduziert. Muttersprachliche Versorgungsangebote können zudem dazu beitragen, dass sich die zur Versorgung verpflichteten Institutionen nicht um eigene Lösungen bemühen, sondern Patienten mit Migrationshintergrund aus ihrem Einzugsbereich auf solche Spezialangebote verweisen.

\section{Interkulturelle Öffnung von Institutionen}

Wir benötigen die Bereitschaft von Kliniken und ambulanten wie auch komplementären Versorgungsstrukturen, sich um Patienten aus anderen Kulturen zu kümmern. Gerade die Menschen mit Migrationshintergrund, die mit dem Medizinsystem in Deutschland wenig vertraut sind und bei denen Verständigungsprobleme (das sind im Übrigen nicht nur sprachliche) bestehen, bedürfen besonderer Beachtung und angemessener Hilfe. Dafür ist interkulturelle Kompetenz aller an der Behandlung beteiligten Berufsgruppen gefordert. Interkulturelle Kompetenz kann als eine Komponente der sozialen Kompetenz definiert werden. Wahrnehmen, Urteilen und Handeln sind immer auch kulturell bedingt [12]. Interkulturelle Kompetenz ist das Resultat eines Entwicklungs- und Lernprozesses, der eine empathische, auf gegenseitiger Wertschätzung getragene Kommunikation und Kooperation auch unter erschwerenden Bedingungen wie Dolmetscherbeteiligung und (anfänglicher) kultureller Fremdheit ermöglicht. Zusätzlich ist die Vermittlung von Kenntnissen über Kultur, Symptomausdruck, Krankheitsmodelle bis hin zu pharmakokinetischen Besonderheiten bestimmter Ethnien erforderlich. Das Gefühl der Fremdheit in der therapeutischen Beziehung kann eine negative Übertragung und/oder Gegenübertragung auslösen. Selbstkritische Reflexion der eigenen blinden Flecken ist Voraussetzung, diese zu überwinden.

\section{Arbeit mit Sprach- und Kulturmittlern (Dolmetschern)}

Um für Patienten mit geringen Kenntnissen der deutschen Sprache eine angemessene Behandlung gewährleisten zu können, müssen gut organisierte und preiswerte Dolmetscherdienste verfügbar sein. Dabei kann es sich z.B. um Gemeindedolmetscher- 
dienste handeln, es sind aber in größeren Kliniken auch Dolmetscherdienste von bilingualen Mitarbeitern denkbar. Der Einsatz von Sprach- und Kulturmittlern sollte routinemäßig dann erfolgen, wenn sprachliche Verständigung nicht ausreichend gewährleistet ist. Es gibt mittlerweile Leitfäden für professionellen Einsatz von Sprach- und Kulturmittlern, die nicht nur einmalig - z. B. bei der stationären Aufnahme - zugezogen werden sollten, sondern sich auch eignen, psychiatrische und psychotherapeutische Behandlungen kontinuierlich zu begleiten. Einfache Regeln helfen hier, Missverständnisse zu vermeiden und die Möglichkeiten, die in einer dolmetschergestützten Behandlung liegen, nutzen zu können [13].

\section{Sprache als Spezialisierung?}

Eine aktuelle Entwicklungsrichtung der Psychiatrie ist zunehmende Spezialisierung. Stationskonzepte werden diagnosebezogen entwickelt, Manuale und Behandlungspfade richten sich im Sinne einer Evidenzbasierung nach erprobten Therapierichtungen, für die zunehmend auch spezielle Ausbildungen oder Zusatzqualifikationen erforderlich sind. Es ist schwer vorstellbar, dass muttersprachliche Behandler alle für eine differenzierte psychiatrische und psychotherapeutische Behandlung notwendigen Curricula absolvieren. Vielmehr erscheint es sinnvoll, in die Behandlungspfade Hinweise auf den Einsatz von Dolmetschern aufzunehmen und dafür Sorge zu tragen, dass testpsychologische Verfahren und Therapiemanuale in den häufigen Sprachen zur Verfügung stehen.

\section{Der Stellenwert von Migrationsbeauftragten}

Die interkulturelle Öffnung von medizinischen Institutionen und insbesondere psychiatrisch-psychotherapeutischen Kliniken sollte vorangebracht werden durch die Etablierung von Migrations- bzw. Integrationsbeauftragten [14], die für die jeweilige Situation der Kliniken geeignete Lösungen und Konzepte erarbeiten und die Umsetzung dieser Konzepte begleiten und überprüfen. Mitarbeiter sollten Fortbildungen in interkultureller Kompetenz erhalten. Dies schließt auch patientenorientierte Fallvorstellungen und interkulturelle Balint-Arbeit ein. Um eine Nachhaltigkeit für die interkulturelle Öffnung zu erreichen, ist es erforderlich, dass die Geschäftsführungen solche Konzepte einrichten (top-down) und gleichzeitig durch Vermittlung der Migrationsbeauftragten die Mitarbeiter der Basis geschult und interessiert werden (bottom-up). Dabei kann die gezielte Einstellung von Mitarbeitern mit Migrationshintergrund zur kulturellen Sensibilisierung der Institutionen beitragen.

\section{Zusammenfassung}

Das Kontra in dieser Debatte meint nicht, dass muttersprachliche Behandler überflüssig sind. Bei der Entwicklung von Konzepten, eine bessere Integration von Patienten mit Migrationshintergrund in die Routineabläufe zu erreichen, können muttersprachliche Behandler eine wichtige Rolle spielen. Allerdings kann die psychiatrische Versorgung für Menschen mit Migrationshintergrund nur über kulturelle Sensibilität aller Mitarbeiter in den multiprofessionellen und multiethnischen Teams verbessert werden. Wir benötigen keine Spezialangebote, die ausschließlich auf muttersprachliche Therapeuten und einzelne Ethnien ausgelegt sind, sondern die generelle Öffnung des medizinischen Sys- tems und das Interesse aller Mitarbeiter an einer angemessenen Behandlung auch der Patienten aus anderen Kulturen. Es geht darum, in der Begegnung mit den Patienten aus anderen Kulturen nicht nur das Fremde im anderen zu sehen, sondern auch die eigene Haltung und Einstellung zu reflektieren und die Kultur der Institutionen so zu verändern, dass sich alle Patienten willkommen und geachtet fühlen $[15,16]$. Es gilt, eine Haltung zu entwickeln, die es auch einheimischen Therapeuten - ggf. unter Dolmetschereinsatz - ermöglicht, eine von gegenseitigem Verstehen getragene Beziehung aufzubauen und zu entwickeln [17].

\section{Literatur}

1 Kirmayer LJ, Groleau D, Guzder J et al. Cultural consultation: a model of mental health service for multicultural societies. Can J Psychiatry 2003; 48: 145-153

2 Tantam D. Therapist-patient interactions and expectations. In: Bhugra D, Bhui K, eds. Textbook of Cultural Psychiatry. Cambridge: Cambridge University Press; 2007: 379-387

3 Mezzich JE, Caracci G, Fabrega $\mathrm{H}$ et al. Cultural Formulation Guidelines. Transcultural Psychiatry 2009; 46: 383-405

4 Kahraman B. Therapeutische Beziehung und Kultur. In: Kahraman B. Die kultursensible Therapiebeziehung. Gießen: Psychosozial Verlag; 2008: 59-76

5 Erim-Frodermann Y, Aygün S, Senf W. Türkeistämmige Migranten in der psychotherapeutisch-psychosomatischen Ambulanz. In: Heise T, Schuler J, Hrsg. Transkulturelle Beratung, Psychotherapie und Psychiatrie in Deutschland. Berlin: Verlag für Wissenschaft und Bildung; 2000: 157-169

6 Schouler-Ocak M. Regelversorgungseinrichtung - PatientInnen türkischer Herkunft in der Institutsambulanz des Niedersächsischen Landeskrankenhauses Hildesheim. In: Heise T, Schuler J, Hrsg. Transkulturelle Beratung, Psychotherapie und Psychiatrie in Deutschland. Berlin: Verlag für Wissenschaft und Bildung; 2000: 81-89

7 Gün AK. Interkulturelle Öffnung in den Institutionen der Gesundheitsdienste. In: Erim Y, Hrsg. Klinische Interkulturelle Psychotherapie. Ein Lehr- und Praxisbuch. Stuttgart: Kohlhammer; 2009: 118-137

8 Özbek T, Wohlfart E. Der transkulturelle Übergangsraum - ein Theorem und seine Funktion in der transkulturellen Psychotherapie am ZIPP. In: Wohlfart E, Zaumseil M, Hrsg. Transkulturelle Psychiatrie - interkulturelle Psychotherapie. Interdisziplinäre Theorie und Praxis. Heidelberg: Springer; 2006: 169-175

9 Erim Y, Kahraman S. „Ich habe noch vier Embryos in Istanbul“: Muttersprachliche Gruppenpsychotherapie mit Migrantinnen aus der Türkei. Psychotherapie im Dialog 2010; 11: 346-351

10 Koch E, Hartkamp N, Siefen RG et al. Patienten mit Migrationshintergrund in stationär-psychiatrischen Einrichtungen - Pilotstudie der Arbeitsgruppe „Psychiatrie und Migration“ der Bundesdirektorenkonferenz. Nervenarzt 2008; 79: 328-339

11 Statistisches Bundesamt 2006. Leben in Deutschland - Ergebnisse des Mikrozensus 2005

12 Grosch H, Leenen WR. Bausteine zur Grundlegung interkulturellen Lernens. In: Bundeszentrale für politische Bildung, Hrsg. Interkulturelles Lernen. Arbeitshilfen für die politische Bildung. Bonn: 1998: 29-46

13 Morina N, Maier T, Schmid Mast M. Lost in Translation? Psychotherapie unter Einsatz von Dolmetschern. Psychother Psych Med 2010; 60: 104-110

14 http://www.bundesregierung.de/Content/DE/Artikel/IB/Artikel/The men/Gesellschaft/Gesundheit/2009-09-01-empfehlungen-arbeits kreis-gesundheit.html

15 Tseng WS, Streltzer J. Cultural Competence in Clinical Psychiatry. Washington: American psychiatric Publishing; 2004

16 Alarcon RD. Culture, cultural factors and psychiatric diagnosis: review and projections. World Psychiatry 2009; 8: 131-139

17 Gün AK. Interkulturelle Missverständnisse in der Psychotherapie: Gegenseitiges Verstehen zwischen einheimischen Therapeuten und türkeistämmigen Klienten. Freiburg: Lambertus; 2007 